\title{
Comparison of two different models of rehabilitation programs following total knee replacement operations
}

\author{
Rabab S. Zaghlol ${ }^{1} \mathbb{D}$, Sahar S. Khalil ${ }^{1,2^{*}}$ (D), Ahmed M. Attia ${ }^{3}$ (D) and Ghada A. Dawa ${ }^{1}$
}

\begin{abstract}
Background: Total knee replacement operation (TKR) is the treatment of choice in severe knee osteoarthritis (OA). Rehabilitation post-TKR is still not well studied. The aim of this study was to compare between the high-intensity (HI) rehabilitation program and the low-intensity (LI) rehabilitation program following TKR.

Results: At 1 month following the TKR operations, significant improvements were found in the first group compared to the second group in all the measured parameters except for the knee range of motion (ROM). At 3 and 12 months follow-up periods, there were statistically significant differences between both groups in all the evaluated parameters except for the numeric pain rating scale and the knee ROM.

Conclusions: Both high-intensity and low-intensity rehabilitation programs are effective; however, HI program had superior functional gain and patient-reported outcomes compared to the LI program. Moreover, HI group has a long-term functional gain.
\end{abstract}

Keywords: Total knee replacement, Rehabilitation, Exercise

\section{Background}

Large joint osteoarthritis $(\mathrm{OA})$ is among the most prevalent diseases causing pain and disability worldwide [1]. The exact etiology and mechanism of developing knee OA are still unknown; however, several factors may increase the risk its occurrence [2]. Knee OA could be induced due to the disturbance of the equilibrium between the cartilage degeneration and regeneration with aging, causing knee joint pain, stiffness, limping, and even deformity. Joint cartilage cannot be regenerated naturally. Delaying the degenerative process is the mainstay of management [3].

Total knee replacement operation (TKR), also known as total knee arthroplasty (TKA), is the treatment of

\footnotetext{
*Correspondence: drsaharkhalil@yahoo.com

'Rheumatology and Rehabilitation Department, Faculty of Medicine, Zagazig

University, Villat al-Gamaa, Zagazig, Sharqia governorate, Egypt

${ }^{2}$ Physical Medicine and Rehabilitation Department, Dubai Hospital, P.O. 7272,

Dubai, United Arab Emirates

Full list of author information is available at the end of the article
}

choice in severe knee OA, with failed conservative treatments. The frequency of TKR operations in the USA is anticipated to reach more than three million by 2030 [4]. Although TKR reliably reduces pain and improves self-reported function in patients with end-stage, however, the optimum functional capacity and muscle strength are still hard to regain after surgery, which may subject the patients to future persistence of pain and disability [5].

Following TKR, around 60\% decrease in quadriceps strength has been reported, even with early starting of the post-operative rehabilitation [6]. This quadriceps weakness may lead to long-term deficit and impairment of functional performance after TKR surgery $[6,7]$. There is a high positive correlation between quadriceps muscle deficits and the continued functional limitations. Hence, interventions are needed to be initiated from the early post-operative phase in order to improve the postoperative quadriceps strength, and this is very important to increase the beneficial outcomes post-TKR [8].

\section{Springer Open}

(c) The Author(s). 2020 Open Access This article is licensed under a Creative Commons Attribution 4.0 International License, which permits use, sharing, adaptation, distribution and reproduction in any medium or format, as long as you give appropriate credit to the original author(s) and the source, provide a link to the Creative Commons licence, and indicate if changes were made. The images or other third party material in this article are included in the article's Creative Commons licence, unless indicated otherwise in a credit line to the material. If material is not included in the article's Creative Commons licence and your intended use is not permitted by statutory regulation or exceeds the permitted use, you will need to obtain permission directly from the copyright holder. To view a copy of this licence, visit http://creativecommons.org/licenses/by/4.0/. 
According to Bade and colleagues, most of the current TKR rehabilitation programs are not fully effective in regaining the optimal muscle strength to normal levels [9].

The aim of this study was to assess the clinical outcomes of high-intensity (HI) rehabilitation program compared to a lower intensity (LI) traditional rehabilitation program following TKR.

\section{Methods}

This study is a randomized controlled study; it included patients who had undergone unilateral cemented TKR through medial parapatellar approach for primary OA and assigned to have post-discharge rehabilitation. Forty patients were randomly recruited from the outpatient clinic of orthopedic department of a big tertiary hospital between January 2017 and June 2018. They were randomly allocated into two groups, 20 patients each. The first group was assigned to follow a high-intensity (HI) rehabilitation program, and the second group (control) was assigned to follow a conventional low-intensity (LI) rehabilitation program. Randomization was conducted through 1:1 random allocation of subjects to each group. Before the step of randomization, legibility criteria were addressed.

The patients were included, if they aged between 50 and 65 years and scheduled for a unilateral TKR operation for primary knee OA. Epidural anesthesia was used in all patients to reduce post-operative pain and allow early rehabilitation.

Exclusion criteria were the following: concurrent medical diseases that could affect rehabilitation progress (e.g., uncontrolled hypertension, uncontrolled diabetes, marked osteoporosis, morbid obesity, advanced malignancy, neurological disease, and decompensated cardiovascular diseases), advanced contra-lateral knee OA with pain score greater than $4 / 10$ with movement, or other lower extremity conditions that cause functional impairment, pacemaker, epilepsy, concurrent orthopedic intervention during surgery that could interfere with outcomes (e.g., collateral or cruciate ligaments repair), and post-operative complication (e.g., pulmonary embolism, heart attack, knee infection, deep venous thrombosis, and problems with scar healing).

Preoperatively, each patient was evaluated by taking full history, body mass index (BMI), clinical examination of the limb and knee joint, functional performance measures, numeric pain rating scale (NPRS), and the Oxford knee score (OKS) [10].

During the hospital stay, the patient received physiotherapy once daily with routine post-operative protocol by a trained physiotherapist. Both groups had initiated their assigned program just post-hospital discharge, and a specified printed form of a unified home exercise program (HEP) was given to each patient to be performed on a daily basis. This program consisted of exercises designed to regain the strength of the operated limb muscles and to improve its mobility.

Patients of both groups had the following common elements in their rehabilitation programs: the usage of neuromuscular electrical stimulation (NMES) to activate the quadriceps muscle directly, patellofemoral joint mobilization, passive knee range of motion (ROM) exercises, scar mobility, cycling for range of motion, lower extremity flexibility exercises, strengthening exercises for the quadriceps, calf and hamstrings, and functional and gait training.

Large flexible self-adhesive electrodes were placed on quadriceps muscle (proximally and distally on the belly of the muscles while the patient is sitting on a chair with the knee $60^{\circ}$ flexion). NMES from the portable electrical stimulator with the following parameters, the amplitude was set to the maximal intensity tolerated by the patient. The stimulator was set to deliver a biphasic current: current frequency $50 \mathrm{~Hz}$, pulse duration $250 \mu \mathrm{s}$, rampup time $3 \mathrm{~s}$, and on-off ration 6:10. Fifteen repetitions were performed during each session.

Group I patients were assigned to perform HI progressive resistance exercise for the operated lower extremity muscle groups adopted from a previously published study done by Bade and co-workers in [11]. The high intensity program was divided into 4 phases:

Phase 1 (weeks 0-2) consisted of a group of simple exercises like heel slide, squats, active knee and ankle ROM exercises, gait training, and neuromuscular electrical stimulation for quadriceps muscle; the criteria of progression to phase 2 are the ability to complete two sets of the designed exercises with eight repetitions without feeling fatigued with NPRS less than 5 at rest and knee ROM more than $15-80^{\circ}$.

Phase 2 (weeks 0-4) consisted of phase 1 exercises in addition to active straight leg raise, hamstrings and calf muscles stretching, and sit-to-stand exercises; the criteria of progression to phase 2 are ability to completing two sets of the designed exercises with eight repetitions without feeling fatigued with NPRS less than 5 at rest and knee ROM more than $15-90^{\circ}$. Moreover, in this phase, resistive exercises are initiated through weights strapped to the ankle joint. After reaching the optimal ankle weight without inducing any fatigue, we gradually shift the patient to machine resistive and strengthening training.

Phase 3 (week 2-12) consists of progression in the intensity of stage 2 exercises, like single-leg calf press, stair climbing (stepping) exercises, tilt board squats, and wall slides knee flexion. Additionally, in this stage progression in resistive and strengthening exercises as tolerated, NMES is applied if there is still quadriceps inhibition, 
weakness, and/or pain. The criteria of progression to phase 4 are ability to completing 2 sets of the designed exercises with eight repetitions without feeling fatigued with NPRS less than 3 at rest and knee ROM more than $10-100^{\circ}$.

Finally, stage 4 (week 6-12): during this stage, there was progression of stage 3 exercises program and initiation of eccentric resistive exercises; these eccentric workouts were performed either through some exercises like seated single-leg knee eccentric flexion and extension, eccentric single-leg calf press, agility exercises: side-shuffle, backward walking, or through using of the weight machine.

Group II (control) patients were assigned to follow a standard low-intensity (LI) rehabilitation program [12]. The LI rehabilitation program was similar to the highintensity program in the first two stages; however, in stage 3 , there was a very gradual initiation of strengthening exercises with the same progression criteria of $\mathrm{HI}$ program, and in stage 4, there was a progression of strengthening exercises as tolerated without any introduction of eccentric resistive exercises.

Both rehabilitation programs were initiated within 1 week post-hospital discharge. The total number of visits was 24 visits over 12 weeks (twice per week) which were scheduled for both groups. Each training session was planned to last for $45-50 \mathrm{~min}$. Thus, the two groups had similar treatment frequency over these 12 weeks. In both groups, therapy was done by the same physiotherapist who experienced in TKR rehabilitation.

In both groups, exercises were advanced, based on attainment of determined milestones as well as the treatment tolerance. Moreover, the exercises were advanced as scheduled, except if the patient had 2 or more of the following findings: an increase of 2 or more points in the resting NPRS, muscle soreness for two or more hours after the last session, a reduction in knee $\mathrm{ROM}$ by $5^{\circ}$ or more, an increase in knee joint swelling of $2 \mathrm{~cm}$ or more, or decreased walking distance from the last session. Hence, the escalation in the session intensity of the following session was accustomed to allow recovery. Nevertheless, if the only finding was muscle soreness lasting 2 to $24 \mathrm{~h}$, we modified the exercises targeting the sored muscle.

\section{Outcomes}

Patients were assessed before the operation and then after 1,3 , and 12 months post-operatively by one investigator, who was blinded of the group assignment. The 12-month assessment period was chosen in order to attain an adequate follow-up period, as patients recovering from TKR usually plateau in both strength and functional gains by 12 months post-operative [13]. The following parameters were used as outcome measures:
1. Numeric pain rating scale (NPRS): Which is an 11point verbal pain rating scale with zero represented no pain and 10 represented the worst pain imaginable. Patients were asked to rate their resting pain during the past $48 \mathrm{~h}$ [14].

2. Knee range of motion (ROM): Active knee ROM was measured using goniometer.

3. Functional performance measures: Measures of functional performance included Stair Climbing Test (SCT), timed up and go test (TUG), and 6 -min walk test $(6 \mathrm{MW})$. The SCT is used to assess the time (in seconds) needed by the subject to ascend and descend a stair flight $(18 \mathrm{~cm}$ step height, 12 steps). The SCT is used to test the physical ability to ascend and descend the stairs, in addition to balance and lower limb strength while using a specific number of stair steps [15]. The TUG is used to measure the mobility and short distant walking along with assessing the power. During this test estimates, the time (in seconds) needed to get-up from an armchair, walk $3 \mathrm{~m}$, go around, and come back to sit in the same chair without any assistance except for walking aid if required [16]. The $6 \mathrm{MW}$ is used to evaluate the capability to walk for considerably longer distances by assessing the maximal total distance walked by an individual at free speed over $6 \mathrm{~min}$ on an on a flat indoor floor [17].

4. Oxford Knee Score (OKS): It was developed to be a simple patient-administered method to evaluate pain and function after TKR operations [10]. OKS includes 12-item patient-reported measure containing questions regarding his pain and function. It consists of a 7-item subscale that measures pain, and 5-item subscale that measures function. Each component involves five potential responses that are scored from zero to 4 . The final score ranges between zero (worst score) and 48 (best score) [18].

5. Adherences to therapy:

a) Attendance adherence: Attendance at the training sessions was collected by the physical therapist delivering the training in a specified log.

b) Home exercise program adherence was recorded via a self-reported exercise log that includes the number of days they did the entire HEP.

c) Physical therapist adherence to the specified program: The physiotherapist filled in a detailed sheet in order to register the amount, duration, and type of treatments given. If the therapy was not conducted according to the specified program, it will not be counted. 


\section{Statistical analysis}

The collected data throughout history, clinical examination, and questionnaires were computerized and statistically analyzed using SPSS program "Statistical Package for Social Science" version 16.0. Suitable statistical tests were chosen and used effectively. The differences were considered statistically significant when $p$ value $\leq 0.05$.

\section{Results}

No differences were found between the two groups regarding all the evaluated variables at the baseline including age, BMI, functional performance measures, knee range of motion, degree of pain, and OKS score $(p$ $>0.05)$ as shown in Table 1.

From Table 2, after 1 month of the TKR operation, we found significant improvements $(p \leq 0.05)$ in group I patients compared to group II in pain and all the other measured parameters, except for the knee ROM. The HI group performed $10.3 \mathrm{~s}$ faster on the SCT (Fig. 1), $4.8 \mathrm{~s}$ faster on the TUG (Fig. 2) and walked $46.5 \mathrm{~m}$ farther on the 6MW (Fig. 3).

At 3 months following TKR, the HI group performed $2.5 \mathrm{~s}$ faster on the SCT (Fig. 1), $1.5 \mathrm{~s}$ faster on the TUG (Fig. 2), and walked $57.5 \mathrm{~m}$ farther on the 6MW (Fig. 3), compared to the control group. Moreover, we found a significant improvement in group I compared to group II in the OKS. However, there were no significant differences between groups in the NPRS and knee ROM as shown in Table 3.

Twelve months following TKR, still, there were statistically significant differences in patients of group I compared to group II in all the evaluated functional and clinical parameters (SCT, TUG, 6MW, OKS). HI group performed $4.2 \mathrm{~s}$ faster on the SCT (Fig. 1), $2.7 \mathrm{~s}$ faster on the TUG (Fig. 2), and walked $37.5 \mathrm{~m}$ farther on the $6 \mathrm{MW}$ (Fig. 3), compared to the control group. There were no significant differences between both groups in the NPRS and knee ROM as shown in Table 4.

Regarding adherence to the specified therapy plan, no statistically significant differences between the two groups were found in either the patients' adherence to sessions' attendance and home exercise program or the physical therapist adherence to the assigned program $(p$ $>0.05$ ) (Table 5).

During the follow-up time, only two patients had trivial problems. In the HI group, one patient experienced low back pain after the first rehabilitation session, which was completely relieved with cold application. In the control group, two patients in group I and 3 patients in group II experienced exacerbated knee swelling during the course of rehabilitation, but all were minimal swellings $(<2 \mathrm{~cm})$. However, these conditions did not affect their program.

\section{Discussion}

The purpose of this study was to compare between the high-intensity $(\mathrm{HI})$ rehabilitation program and the lowintensity (LI) rehabilitation program following total knee replacement operation.

Until the current time, there are no internationally accepted or widely agreed and implemented clinical protocols existing to reliably determine the optimal rehabilitation following TKR, and there are many debates regarding this issue. Adding to that, the paucity

Table 1 Preoperative characteristics of both groups

\begin{tabular}{|c|c|c|c|}
\hline Variable & HI group $(\boldsymbol{n}=20)$ & LI group $(\boldsymbol{n}=20)$ & $\boldsymbol{P}$ value \\
\hline Age, (years) mean $\pm S D$ & $58.1 \pm 3.9$ & $58.1 \pm 3.8$ & 0.96 \\
\hline $\mathrm{BMI}, \mathrm{kg} / \mathrm{m}^{2}$, mean $\pm \mathrm{SD}$ & $30.3 \pm 1.7$ & $30.1 \pm 1.6$ & 0.63 \\
\hline Stair climbing test (seconds), mean \pm SD & $18.3 \pm 1.6$ & $18.2 \pm 1.7$ & 0.93 \\
\hline Timed up and go test (seconds), mean \pm SD & $8.8 \pm 0.7$ & $8.8 \pm 1.1$ & 1.00 \\
\hline 6-min walk test ( minutes), mean $\pm S D$ & $476 \pm 17.5$ & $475.5 \pm 16.7$ & 0.92 \\
\hline \multicolumn{4}{|l|}{ Knee flexion, degree } \\
\hline Mean \pm SD & $114.2 \pm 7.5$ & $16.1 \pm 7.8$ & 0.25 \\
\hline Range & $106-122$ & $110-126$ & \\
\hline \multicolumn{4}{|l|}{ Knee extension, degree } \\
\hline Mean \pm SD & $-1.5 \pm 2.5$ & $-0.7 \pm 2.7$ & 0.28 \\
\hline Range & $-4-2$ & $-3-2$ & \\
\hline \multicolumn{4}{|l|}{ NPRS } \\
\hline Mean \pm SD & $5.1 \pm 1.2$ & $4.9 \pm 1.2$ & 0.60 \\
\hline Range & $2-7$ & $2-7$ & \\
\hline OKS, mean \pm SD & 19.56 .7 & $20.3 \pm 7.1$ & 0.72 \\
\hline
\end{tabular}

BMI body mass index, NPRS numeric pain rating scale, OKS Oxford Knee Score, $H I$ high-intensity rehabilitation group, $L I$ low-intensity rehabilitation group $P$ value $\leq 0.05$ : significant 
Table 2 Post-operative outcome measures of both groups at 1 month

\begin{tabular}{|c|c|c|c|}
\hline Time/variable & HI group $(\boldsymbol{n}=20)$ & LI group $(\boldsymbol{n}=20)$ & $\boldsymbol{P}$ value \\
\hline Stair climbing test, seconds, mean \pm SD & $25.8 \pm 2.1$ & $36.1 \pm 2.6$ & $0.000^{*}$ \\
\hline Timed up and go test (seconds), mean \pm SD & $8.1 \pm 1.1$ & $12.9 \pm 1.3$ & $0.000^{*}$ \\
\hline 6-min walk test (minute), mean $\pm S D$ & $407 \pm 35.7$ & $360.5 \pm 27.6$ & $0.000^{*}$ \\
\hline \multicolumn{4}{|l|}{ Knee flexion (degree) } \\
\hline Mean \pm SD & $86.6 \pm 10.6$ & $82.5 \pm 11.6$ & 0.19 \\
\hline Range & $75-98$ & 70-95 & \\
\hline \multicolumn{4}{|l|}{ Knee extension (degree) } \\
\hline Mean \pm SD & $2.5 \pm 3.4$ & $1.4 \pm 3.39$ & 0.27 \\
\hline Range & $-2-6$ & $-2-7$ & \\
\hline \multicolumn{4}{|l|}{ NPRS during rest } \\
\hline Mean \pm SD & $4 \pm 0.8$ & $5 \pm 1.1$ & $0.03^{*}$ \\
\hline Range & $1-6$ & $2-7$ & \\
\hline $\mathrm{OKS}$, mean $\pm \mathrm{SD}$ & $30.9 \pm 6.6$ & $24.2 \pm 7.5$ & $0.000^{*}$ \\
\hline
\end{tabular}

NPRS numeric pain rating scale, OKS Oxford Knee Score, $H I$ high-intensity rehabilitation group, $L /$ low-intensity rehabilitation group ${ }^{*} P$ value $\leq 0.05$ : significant

and the heterogeneity of most of the researches that investigated the role of physiotherapy post-TKR [19-24].

In the current study, we found at the first month postoperative follow-up a significant improvement of pain in high-intensity exercise group (HI) compared to the lowintensity exercises (LI) group, but no difference was noticed at 3 and 12 months follow-up period. This improvement in pain was surprising, owing to the expected increase of pain with progressive resistive exercises, especially in the post-operative period. However, this could be attributed to the combined effects of implementing both NMES and progressive strengthening exercises to decrease the post-operative quadriceps central activation deficits (CAD), and consequently better quadriceps power and function, which led to improved pain with limb loading and improved functional recovery [25]. The quadriceps muscle plays a crucial role for shock absorption during joint function; thus, its weakness decreases the joint protection capacity and disturbing biomechanics leading to greater pain from the overload and stress on the knee [26]. Moreover, the exercises were accustomed to avoid any muscle soreness or subjective exacerbation of pain. So, the patients have benefited from the progressive increase of their power without exacerbating of pain.

Moreover, the data showed that the high-intensity exercise group had superior functional gain and patientreported outcomes compared to the low-intensity exercise group at 1 and 3 months follow-up periods. Moreover, the high-intensity exercise group continued to demonstrate this gain at the 12 months follow-up period.

A recent systematic review study which has investigated the effect of different post-discharge physiotherapy program on chronic pain post-TKR; they concluded that there is no evidence that any type of the physiotherapy programs is more superior to another on reducing the intensity of chronic pain post-TKR [27].

Our results were consistent with a randomized controlled study done on 2011 by Bade and co-workers [11], compared with the HI program to a lower intensity rehabilitation program in post-TKR patients. The

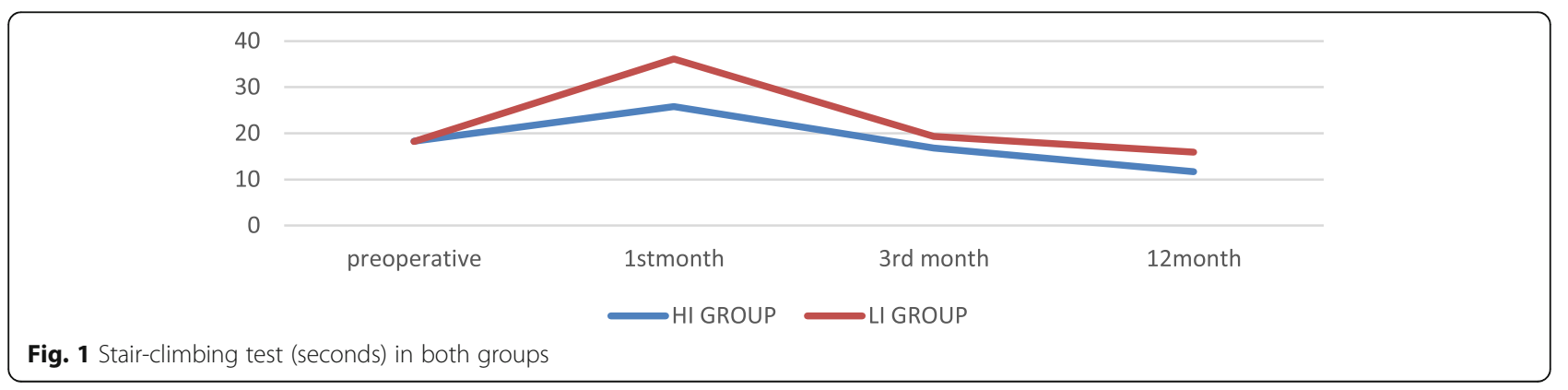




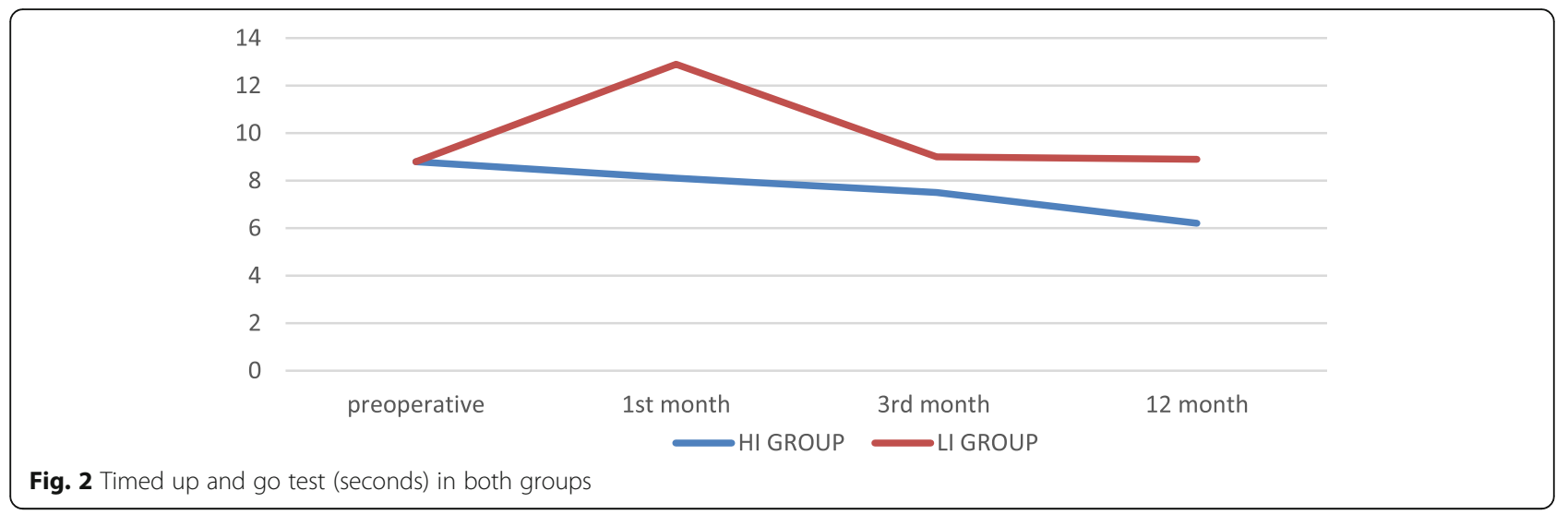

outcomes of therapy included pain scale, ROM, functional evaluation, and measurements of quadriceps muscle power; they concluded that the HI program leads to better strength (short- and long-term) and functional gains compared to the LI rehabilitation program. Moreover, they concluded that the HI program did not result in knee ROM impairment nor any musculoskeletal injuries.

Similarly, Moffet and colleagues [28] compared the intensive functional rehabilitation (IFR) program to the standard care. They concluded that IFR was effective in improving the functional capability after primary TKR in the first year post-surgery. Their results support our argument that intensive rehabilitation improves function after TKR.

Moreover, Petterson and coworkers [29] in their study have compared the progressive strengthening with conventional rehabilitation, and they revealed that the conventional rehabilitation group had less power and functional gain post-TKR at 12 months. They concluded that progressive quadriceps muscle strengthening, with or without the using of neuromuscular electrical stimulation, had enhanced clinical improvement after TKR. Moreover, they concluded that individuals who do not undertake an intensive rehabilitation program following
TKR are clearly at a disadvantage, as the conventional rehabilitation does not yield similar outcomes. Moreover, they concluded that progressive HI rehabilitation program could lead to improved outcomes in this population.

In addition, following knee injury or surgery, the combination of muscle training and neuromuscular electrical stimulation may achieve greater improvement in quadriceps strength when compared with volitional training alone. The effect of the neuromuscular electrical stimulation may be through minimizing quadriceps muscle fibers atrophy after joint damage, thus reducing quadriceps weakness and contradicting the arthrogenic muscular inhibition [30, 31].

In two meta-analyses done to study the effectiveness of post-discharge rehabilitation following TKA, they concluded that following TKA, physiotherapy had induced only short-term improvements in physical function. However, they attributed that to the few numbers of studies used [32, 33].

In contradiction to us, Bade and co-workers in another study conducted on 2017 [34] have concluded from their randomized controlled trial, which also compared the $\mathrm{HI}$ program to the LI program after TKR, that no differences were found between the two groups in muscle

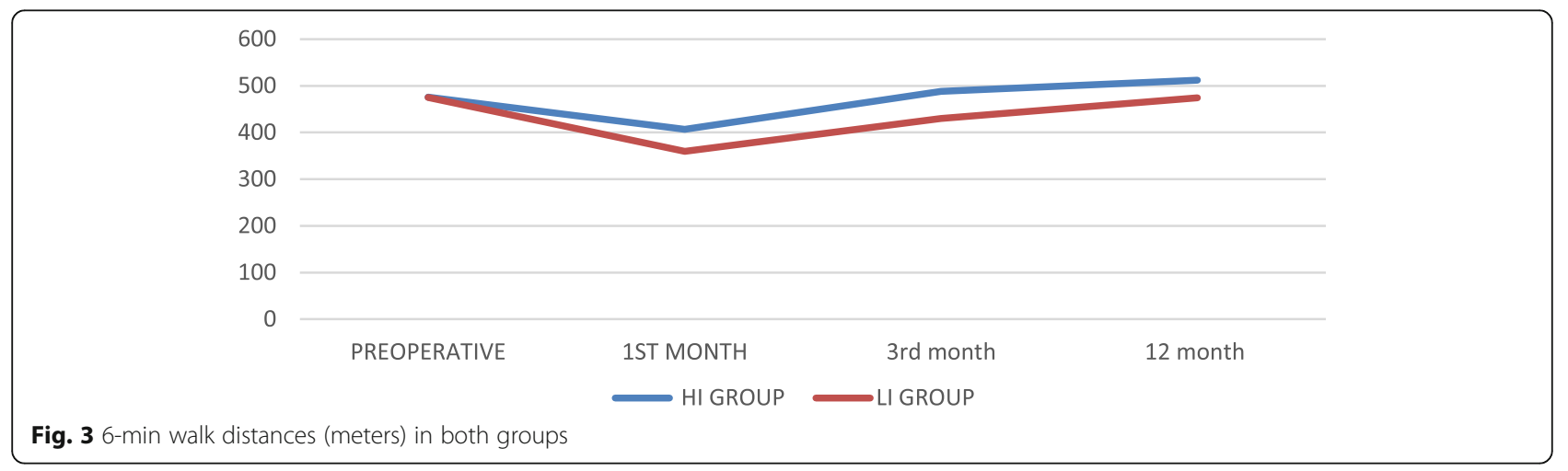


Table 3 Post-operative outcome measures of both groups at 3 months

\begin{tabular}{|c|c|c|c|}
\hline Time/variable & HI group $(\boldsymbol{n}=20)$ & LI group $(\boldsymbol{n}=20)$ & $\boldsymbol{P}$ value \\
\hline Stair climbing test, seconds, mean \pm SD & $16.8 \pm 1.2$ & $19.3 \pm 1.4$ & $0.00^{*}$ \\
\hline Timed up and go test, seconds, mean \pm SD & $7.5 \pm 0.7$ & $9.0 \pm 1.2$ & $0.00^{*}$ \\
\hline 6-minute walk test, minute, mean \pm SD & $488 \pm 46.3$ & $430.5 \pm 58.6$ & $0.00^{*}$ \\
\hline \multicolumn{4}{|l|}{ Knee flexion, degree } \\
\hline Mean \pm SD & $103.3 \pm 10.0$ & $101.1 \pm 10.2$ & 0.46 \\
\hline Range & $(93-114)$ & $(90-112)$ & \\
\hline \multicolumn{4}{|l|}{ Knee extension, degree } \\
\hline Mean \pm SD & $3.35 \pm 2.6$ & $2.45 \pm 2.39$ & 0.21 \\
\hline Range & $(1-6)$ & $(1-5)$ & \\
\hline \multicolumn{4}{|l|}{ NPRS during resting } \\
\hline Mean \pm SD & $3.3 \pm 1.5$ & $3.1 \pm 1.4$ & 0.75 \\
\hline Range & $(1-6)$ & $(1-6)$ & \\
\hline $\mathrm{OKS}$, mean $\pm \mathrm{SD}$ & $32.0 \pm 7.9$ & $30.9 \pm 6.6$ & $0.04^{*}$ \\
\hline
\end{tabular}

NPRS numeric pain rating scale, OKS Oxford Knee Score, $H I$ high-intensity rehabilitation group, $L /$ low-intensity rehabilitation group

*P value $\leq 0.05$ : significant

power, functional gain, and patient-reported outcomes at neither 3 nor 12 months. They attributed the limited efficacy of the HI program than expected to the arthrogenic muscular inhibition of quadriceps muscle in the early post-operative period, which can prevent the

Table 4 Post-operative outcome measures of both groups after 12 months

\begin{tabular}{|c|c|c|c|}
\hline Time/variable & HI group $(\boldsymbol{n}=20)$ & LI group $(\boldsymbol{n}=20)$ & $\boldsymbol{P}$ value \\
\hline \multicolumn{4}{|c|}{ Stair climbing test (seconds) } \\
\hline mean $\pm S D$ & $11.7 \pm 1.2$ & $15.9 \pm 1.2$ & $0.00 *$ \\
\hline Range & $10-14$ & $14-19$ & \\
\hline \multicolumn{4}{|c|}{ Timed up and go test (seconds) } \\
\hline Mean \pm SD & $6.2 \pm 0.8$ & $8.9 \pm 1.1$ & $0.00^{*}$ \\
\hline Range & $5-7$ & $6-12$ & \\
\hline \multicolumn{4}{|c|}{ 6-min walk test (minutes) } \\
\hline Mean \pm SD & $512 \pm 50.5$ & $474.5 \pm 17.1$ & $0.00^{*}$ \\
\hline Range & $380-590$ & $450-500$ & \\
\hline \multicolumn{4}{|c|}{ Knee flexion (degree) } \\
\hline Mean \pm SD & $105.3 \pm 9.07$ & $101.6 \pm 11.1$ & 0.21 \\
\hline Range & $(95-116)$ & $(91-124)$ & \\
\hline \multicolumn{4}{|c|}{ Knee extension (degree) } \\
\hline Mean \pm SD & $3.0 \pm 1.9$ & $2.0 \pm 1.7$ & 0.07 \\
\hline Range & $(1-5)$ & $(1-3)$ & \\
\hline \multicolumn{4}{|l|}{ NPRS } \\
\hline Mean \pm SD & $1.6 \pm 0.5$ & $1.8 \pm 0.4$ & 0.32 \\
\hline Range & $1-2$ & $1-2$ & \\
\hline OKS, mean \pm SD & $40.9 \pm 8.3$ & $33.4 \pm 6.4$ & $0.00^{*}$ \\
\hline
\end{tabular}

NPRS numeric pain rating scale, OKS Oxford Knee Score, $H I$ high-intensity rehabilitation group, $L /$ low-intensity rehabilitation group

${ }^{*} P$ value $\leq 0.05$ : significant quadriceps from being fully activated, hence limits the gain from progressive resistance exercises. Therefore, they recommended future researches to study the level of activation failure and whether the addition of alternative strategies such as neuromuscular electrical stimulation could be more effective in the early post-operative period [34]. Moreover, in consistent with our result, another randomized controlled study has concluded that the using of neuromuscular electrical stimulation has been shown to potentiate the activation of the quadriceps muscle and increase its strength following TKA, even in absence of a supervised physiotherapy program [35].

Both of the following reasons may explain the difference between our results and that of Bade and coworkers [34]. The first potential reason is the use of neuromuscular electrical stimulation in our study to prevent the arthrogenic muscular inhibition of quadriceps muscle in the early post-operative period. The other potential reason is the impact of home exercise program adherence in our study compared to them; as in our study, there was no significant difference between groups, while a significant difference between groups in the adherence to home exercise program was present in their study; (i.e., low-intensity exercises group had completed more home exercise program sessions).

The limitation of the current study is the small number of participants. Moreover, another limitation is that the treating physiotherapist was not be blinded however, proper measures were taken to evade this weakness by blinding both the patients and the assessing physiatrist to the group allocation. Studies with larger number of patients are needed to attain the optimal rehabilitation protocol post-TKR. 
Table 5 Adherence of both the therapist and the patients to the assigned therapy program in both groups

\begin{tabular}{llll}
\hline & HI group $(\boldsymbol{n}=20)$ & LI group $(\boldsymbol{n}=20)$ & $\boldsymbol{P}$ value \\
\hline Patient attendance adherence, mean \pm SD & $97.9 \pm 8.3$ & $96.9 \pm 7.1$ & 0.68 \\
Home exercise adherence, mean \pm SD & $96.7 \pm 5.3$ & $95.7 \pm 3.5$ & 0.48 \\
Therapist adherence, mean \pm SD & $96.7 \pm 4.3$ & $96.2 \pm 7.8$ & 0.80 \\
\hline
\end{tabular}

$P$ value $\leq 0.05$ : significant

\section{Conclusion}

The early implementation of a high-intensity rehabilitation program following total knee replacement operations had resulted in greater short- and long-term functional gain compared to the lower intensity rehabilitation program, so it is advisable to be implemented in order to augment the functional outcomes post-TKR surgeries.

\section{Abbreviations}

OA: Osteoarthritis; TKR: Total knee replacement; TKA: Total knee arthroplasty; OKS: Oxford knee score; HI: High intensity; LI: Low intensity; HEP: Home exercise program; SCT: Stair climbing test; TUG: Timed up and go test; 6MW: 6-min walk test; NPRS: Numeric pain rating scale; HEP: Home exercise program; ROM: Range of motion; NMES: Neuromuscular electrical stimulation; BMI: Body mass index; IFR: Intensive functional rehabilitation

\section{Acknowledgements}

None

\section{Authors' contributions}

All authors have contributed to designing the study, collecting and analyzing, interpretation of data, and preparing and revising the manuscript. Design of the study: RS, SK, AM, and GA. Recruitment of patients: RS, SK, AM, and GA. Data collection: RS, SK, AM, and GA. Randomizing: RS and SK. Assessment: RS, SK, and GA. Statistical analysis and data interpretation: RS, SK, AM, and GA. Manuscript preparation: RS, SK, AM, and GA. Manuscript revision: $\mathrm{RS}, \mathrm{SK}, \mathrm{AM}$, and GA. All co-authors have approved the final version of the manuscript.

\section{Funding}

This study has no funding sources.

\section{Availability of data and materials}

The data will be available upon request.

\section{Ethics approval and consent to participate}

The study protocol was approved by the Zagazig University Hospital Ethics Committee with reference number ZU-IRB\#6198. The study was conducted in accordance with the ethical standards of Helsinki declaration. Informed written consent has been obtained from all subjects involved in the study prior to participation.

\section{Consent for publication}

Not applicable

\section{Competing interests}

Authors have no conflict of interest.

\section{Author details}

${ }^{1}$ Rheumatology and Rehabilitation Department, Faculty of Medicine, Zagazig University, Villat al-Gamaa, Zagazig, Sharqia governorate, Egypt. ${ }^{2}$ Physical Medicine and Rehabilitation Department, Dubai Hospital, P.O. 7272, Dubai, United Arab Emirates. ${ }^{3}$ Orthopedic Department, Faculty of Medicine, Zagazig University, Zagazig, Sharqia governorate, Egypt.
Received: 16 June 2020 Accepted: 12 July 2020

Published online: 22 December 2020

\section{References}

1. Hunter DJ (2011) Lower extremity osteoarthritis management needs a paradigm shift. Br J Sports Med 45:283-288

2. Palmer K (2012) Occupational activities and osteoarthritis of the knee. $\mathrm{Br}$ Med Bull 102:147-170

3. Saoji A, Mahajan S, Deshpande S (2015) Added advantage of ayurvedic management in a series of 100 cases of osteoarthritis of the knee joint: a shared experience between Orthopaedic and Ayurvedic faculty. Egypt Rheumatol Rehabil 42:7-10

4. Kurtz S, Ong K, Lau E, Mowat F, Halpern M (2007) Projections of primary and revision hip and knee arthroplasty in the United States from 2005 to 2030. J Bone Joint Surg Am 89(4):780-785

5. Valtonen A, Pöyhönen T, Heinonen A, Sipilä S (2009 Oct 1) Muscle deficits persist after unilateral knee replacement and have implications for rehabilitation. Phys Ther 89(10):1072-1079

6. Mizner R, Petterson S, Stevens J, Vandenborne K, Snyder-Mackler L (2005) Early quadriceps strength loss after total knee arthroplasty. The contributions of muscle atrophy and failure of voluntary muscle activation. $J$ Bone Joint Surg Am 87:1047-1053

7. Noble P, Gordon M, Weiss J, Reddix R, Conditt M, Mathis K (2005) Does total knee replacement restore normal knee function? Clin Orthop Relat Res:157-165

8. Mizner R, Petterson S, Snyder-Mackler L (2005) Quadriceps strength and the time course of functional recovery after total knee arthroplasty. J Orthop Sports Phys Ther 35(7):424-436

9. Bade M, Kohrt W, Stevens-Lapsley J (2010) Outcomes before and after total knee arthroplasty compared to healthy adults. J Orthop Sports Phys Ther 40(9):559-567

10. Dawson J, Fitzpatrick R, Murray D, Carr A (1998) Questionnaire on the perceptions of patients about total knee replacement surgery. J Bone Joint Surg 80-B:63-69

11. Bade M, Stevens-Lapsley J (2011) Early high-intensity rehabilitation following total knee arthroplasty improves outcomes. J Orthop Sports Phys Ther. 41(12):932-941

12. Mintken P, Carpenter K, Eckhoff D, Kohrt W, Stevens J (2007) Early neuromuscular electrical stimulation to optimize quadriceps muscle function following total knee arthroplasty: a case report. J Orthop Sports Phys Ther. 37:364-371

13. Kennedy D, Stratford P, Riddle D, Hanna SE, Gollish J (2008) Assessing recovery and establishing prognosis following total knee arthroplasty. Phys Ther 88:22-32

14. Childs JD, Piva SR, Fritz JM (2005) Responsiveness of the numeric pain rating scale in patients with low back pain. Spine 30:1331-1334

15. Kennedy DM, Stratford PW, Wessel J, Gollish JD, Penney D (2005) Assessing stability and change of four performance measures: a longitudinal study evaluating outcome following total hip and knee arthroplasty. BMC Musculoskelet Disord 6:3

16. Mathias S, Nayak US, Isaacs B (1986) Balance in elderly patients: the "get-up and go" test. Arch Phys Med Rehabil 67:387-389

17. Balke B (1963) A simple field test for the assessment of physical fitness: rep 63-6. Rep Civ Aeromed Res Inst US:1-8

18. Murray D, Fitzpatrick R, Rogers K, Pandit H, Beard D, Carr A et al (2007) The use of the Oxford hip and knee scores. J Bone Joint Surg Br 89:1010-1014

19. Naylor J, Harmer A, Fransen M, Crosbie J, Innes L (2006) Status of physiotherapy rehabilitation after total knee replacement in Australia. Physiother Res Int 11(1):35-47 
20. Mistry JB, Elmallah RD, Bhave A, Chughtai M, Cherian JJ, McGinn T et al (2016) Rehabilitative guidelines after total knee arthroplasty: a review. J Knee Surg 29(3):201-217

21. Peter WF, Nelissen RG, Vlieland TP (2014) Guideline recommendations for post-acute postoperative physiotherapy in total hip and knee arthroplasty: are they used in daily clinical practice? Musculoskeletal Care 12(3):125-131

22. Oatis CA, Li W, DiRusso JM, Hoover MJ, Johnston KK, Butz MK et al (2014) Variations in delivery and exercise content of physical therapy rehabilitation following total knee replacement surgery: a cross-sectional observation study. Int J Phys Med Rehabil (Suppl 5). https://doi.org/10. 4172/2329-9096.S5-002

23. Lingard EA, Berven S, Katz JN (2000) Management and care of patients undergoing total knee arthroplasty: variations across different health care settings. Arthritis Care Res 13(3):129-136

24. Freburger JK, Holmes GM, Ku L, Cutchin MP, Heatwole-Shank K, Edwards L (2011) Disparities in post-acute rehabilitation care for joint replacement. Arthritis Care Res (Hoboken) 63(7):1020-1030

25. Thomas AC, Stevens-Lapsley JE (2012) Importance of attenuating quadriceps activation deficits after total knee arthroplasty. Exerc Sport Sci Rev 40(2):95-101

26. Hurley MV (1999) The role of muscle weakness in the pathogenesis of osteoarthritis. Rheum Dis Clin N Am 25(2):283-298

27. Wylde V, Dennis J, Gooberman-Hill R, Beswick AD (2018) Effectiveness of postdischarge interventions for reducing the severity of chronic pain after total knee replacement: systematic review of randomised controlled trials. BMJ Open 8(2):e020368

28. Moffet H, Collet J, Shapiro S, Paradis G, Marquis F, Roy L (2004) Effectiveness of intensive rehabilitation on functional ability and quality of life after first total knee arthroplasty: a single-blind randomized controlled trial. Arch Phys Med Rehabil 85:546-556

29. Petterson S, Mizner R, Stevens J, Raisis L, Bodenstab A, Newcomb W et al (2009) Improved function from progressive strengthening interventions after total knee arthroplasty: a randomized clinical trial with an imbedded prospective cohort. Arthritis Rheum 61:174

30. Snyder-Mackler L, Ladin Z, Schepsis A, Young J (1991) Electrical stimulation of the thigh muscles after reconstruction of the anterior cruciate ligament: effects of electrically elicited contraction of the quadriceps femoris and hamstring muscles on gait and on strength of the thigh muscles. J Bone Joint Surg Am 73(7):1025-1036

31. Stevens J, Mizner R, Snyder-Mackler L (2004) Neuromuscular electrical stimulation for quadriceps muscle strengthening after bilateral total knee arthroplasty: a case series. J Orthop Sports Phys Ther. 34(1):21-29

32. Minns Lowe CJ, Barker KL, Dewey M, Sackley CM (2007) Effectiveness of physiotherapy exercise after knee arthroplasty for osteoarthritis: systematic review and meta-analysis of randomised controlled trials. Bmj 335(7624):812

33. Artz N, Elvers KT, Lowe CM, Sackley C, Jepson P, Beswick AD (2015) Effectiveness of physiotherapy exercise following total knee replacement: systematic review and meta-analysis. BMC Musculoskelet Disord 16:15

34. Bade M, Struessel T, Dayton M, Foran J, Kim R, Miner T et al (2017) Early high-intensity versus low-intensity rehabilitation after total knee arthroplasty: a randomized controlled trial. Arthritis Care Res (Hoboken) 69(9):1360-1368

35. Levine M, McElroy K, Stakich V, Cicco J (2013) Comparing conventional physical therapy rehabilitation with neuromuscular electrical stimulation after TKA. Orthopedics. 36(3):319-324

\section{Publisher's Note}

Springer Nature remains neutral with regard to jurisdictional claims in published maps and institutional affiliations.

\section{Submit your manuscript to a SpringerOpen ${ }^{\circ}$ journal and benefit from:}

- Convenient online submission

- Rigorous peer review

- Open access: articles freely available online

- High visibility within the field

- Retaining the copyright to your article

Submit your next manuscript at $\boldsymbol{\nabla}$ springeropen.com 